\title{
Descripción preliminar sobre el desarrollo oocitario del mapará Hypophthalmus marginatus (Siluriformes: Pimelodidae) en el río Ariari, municipio de Puerto Rico, Meta
}

\author{
Preliminary description on the oocyte development of the mapara \\ Hypophthalmus marginatus (Siluriformes: Pimelodidae) in the Ariari \\ river, municipality of Puerto Rico, Meta
}

\section{Descrição preliminar sobre o desenvolvimento oocitário do mapará Hypophthalmus marginatus (Siluriformes: Pimelodidae) no rio Ariari, município de Porto Rico, Meta}

\author{
Jonathan F. Villamil-Rodríguez ${ }^{1 *}$; José A. Rodríguez-Pulido ${ }^{*}$ \\ 1 Lic. en Prod. Agrop, Esp, MSc, Instituto de Acuicultura, Facultad de Ciencias Agropecuarias y Recursos Naturales, \\ Universidad de los Llanos, Villavicencio, Colombia. \\ 2 Biol, MSc, (c)PhD, Profesor Universidad de los Llanos, Facultad de Ciencias Básicas e Ingeniería, Programa de Biología, \\ Villavicencio, Colombia. \\ * Grupo de Investigación sobre Reproducción y Toxicología de Organismos Acuáticos - GRITOX. Instituto de Acuicultura, \\ Universidad de los Llanos, Villavicencio, Colombia. \\ Email: jonathan01.Ipa@gmail.com
}

Recibido: 27 de julio de 2018

Aceptado: 04 de diciembre de 2018

\begin{abstract}
Resumen
El mapará Hypophthalmus marginatus es un bagre planctófago perteneciente a la familia Pimelodidae y es nativo de las cuencas más importantes de América del Sur. Uno de los requisitos esenciales para mantener el estoque pesquero y ampliar la oferta de especies cultivables es el estudio de los ciclos reproductivos en peces nativos, el cual contribuye significativamente al conocimiento biológico de las especies. El objetivo del presente estudio, pretende abordar de forma preliminar la caracterización del desarrollo oocitario de H. marginatus. Fueron analizadas las características histológicas de las gónadas de 17 hembras sexualmente maduras capturadas en el río Ariari (Puerto Rico, Meta), encontrando todos los tipos celulares del linaje germinativo, permitiendo establecer que es una especie del tipo asincrónico con desoves parciales.
\end{abstract}

Palabras clave: oogénesis; planctófago; reproducción.

\begin{abstract}
The mapara Hypophthalmus marginatus is a planktophagous catfish belonging to the family Pimelodidae and is native to the most important basins in South America. One of the essential requirements to maintain the fishing stock and expand the
\end{abstract}


supply of cultivable species is the study of reproductive cycles in native fish, which contributes significantly to the biological knowledge of the species. The objective of the present study, intends to approach in a preliminary way the characterization of the oocyte development of $\mathrm{H}$. marginatus. The histological characteristics of the gonads of 17 sexually mature females captured in the Ariari River (Puerto Rico, Meta) were analyzed, finding all cell types of the germinative lineage, allowing to establish that it is an asynchronous specie with partial spawning.

Keywords: oogenesis; planktophagous; reproduction.

\begin{abstract}
Resumo
O mapará Hypophthalmus marginatus é um bagre planctófago pertencente à familia Pimelodidae e é nativo das bacias mais importantes da América do Sul. Um dos pré-requisitos essenciais para manter o estoque pesqueiro e ampliar a oferta de espécies cultivadas é o estudo dos ciclos reprodutivos em peixes nativos, a qual contribui significativamente ao conhecimento biológico das espécies. O objetivo deste estudo foi abordar de forma preliminar a caracterização do desenvolvimento oocitario de H. marginatus. Foram analisadas as características histológicas das gônadas de 17 fêmeas sexualmente maduras capturadas no rio Ariari (Puerto Rico, Meta) encontrando todos os tipos celulares da linhagem germinativa, permitindo estabelecer que é uma especie do tipo assincrônico com desovas parciais.
\end{abstract}

Palavras chave: oogênese; planctófago; reprodução.

\section{Introducción}

El grupo más numeroso de vertebrados es el de los peces, al contener alrededor de 28.000 especies, pero es América del Sur, es el que contiene mayor diversidad que los otros continentes, con un número estimado de más de 4.000 especies (Reis, 2013; Nelson et al., 2016). Sin embargo, entre las especies nativas altamente exploradas y con gran proyección para la acuicultura se encuentran los maparás, destacados por ser peces de mediano porte, pertenecientes al orden de los Siluriformes y caracterizados por su amplia distribución en América del Sur, encontrándose en áreas lénticas y lóticas de las cuencas del Orinoco y Amazonas (Alcântara-Neto, 1994; Agostinho et al., 1994; Araújo-Lima y Ruffino, 2004). Mapará es el nombre común dado a cuatro especies del mismo género de la familia Pimelodidae, siendo Hypophthalmus marginatus, Hypophthalmus edentatus, Hypophthalmus fimbriatus e Hypopthalmus oremaculatus (Carvalho y Goulding, 1985; López-Fernándes y Winemiller, 2000; Araújo-Lima y Ruffino, 2004; Ferraris-Jr, 2007) respectivamente. Se caracterizan principalmente por su hábito alimenticio planctófago, lo que los hace diferentes a los demás Siluriformes (Cutrim y Batista, 2005).

Por lo tanto, para que una especie sea incorporada a sistemas confinados, se requiere antes que nada conocer en ambiente natural algunos aspectos relacionados con su trofodinámica y en este caso, su comportamiento reproductivo. En hembras, la oogénesis es mediada por el eje hipotálamo-hipófisis-gónadas, el cual sintetiza y libera gonadotropinas, hormonas moduladoras y esteroides gonadales para llevar a cabo la reproducción (Zohar et al., 2010; Da Silva Ribeiro y Moreira, 2012; Honji y Guimarães, 2017).

Para lograr la culminación del evento reproductivo, debe haber una sincronización con los parámetros ambientales, siendo fotoperiodo, temperatura y pluviosidad los más relevantes (Lowe-McConnell, 1987; Nahagama y Yamashita, 2008; Mylonas et al., 2010). De manera general, el desarrollo de las células de la línea germinativa parte de las oogonias hasta convertirse en oocitos, que inicia con la acumulación de substancias nutricionales hasta incrementar la actividad metabólica por la producción de vitelo, finalizando con la maduración y ovulación (Kagawa, 2013; Hiramatsu et al., 2015).

De acuerdo a las anteriores consideraciones y teniendo en cuenta la escasez de información sobre la oogénesis del género Hypophthalmus en Sudamérica, solamente en dos reportes se ha descrito este proceso de desarroIlo celular, el primero corresponde al estudio de Benedito-Cecilio y Agostinho (1991) en H. edentatus donde se describen todos los tipos celulares, caracterizándola como una especie asincrónica y con desoves parciales. El segundo y más reciente trabajo realizado por HainfeIlner (2015) en $H$. marginatus, quien describe a la especie con un proceso de reposo reproductivo prolongado y de vitelogénesis corta, relacionándola aparentemente con ciclos reproductivos de desove total. Por lo tanto, este trabajo, tiene como objetivo describir el desarrollo oocitario en $H$. marginatus a fin de contribuir no solo en la caracterización de su tipo de reproducción en la Orinoquia colombiana, sino también en aras de ampliar el conocimiento de esta especie promisoria para la piscicultura regional y nacional. 


\section{Materiales y métodos}

\section{Área de estudio}

Para efectos del trabajo de campo, los ejemplares fueron colectados en el río Ariari $\left(3^{\circ} 12^{\prime} 53 N 73^{\circ} 25^{\prime} 11^{\prime \prime} \mathrm{W}\right)$ del municipio de Puerto Rico-Meta (Colombia), el cual presenta una altitud de $216 \mathrm{msnm}$, temperatura de $27^{\circ} \mathrm{C}$ y una precipitación media aproximada de 2827 mm. Para la evaluación histológica de las láminas, el trabajo fue llevado a cabo en el Laboratorio de Reproducción y Crioconservación de Gametos del Instituto de Acuicultura de la Universidad de los Llanos (IALL-UNILLANOS), ubicado en la ciudad de Villavicencio-Meta (Colombia) a una altura de 418 msnm, temperatura promedio de $26^{\circ} \mathrm{C}$, humedad relativa del $75 \%$ y precipitación de $4050 \mathrm{~mm}$.

\section{Material biológico}

Se capturaron 17 hembras de mapará (Hypophthalmus marginatus) con una longitud total (Lt) de 50,74 $\pm 1,38$ y peso promedio de $683,38 \pm 18,38 \mathrm{~g}$ (Figura 1) durante los meses de abril, mayo, septiembre y diciembre del año 2017. En horarios diurnos, fueron extendidas redes de espera de 180 metros de longitud y 2.5 metros de altura, revisiones periódicas se hicieron necesarias para determinar la presencia de los individuos (Benedito-Cecilio y Agostinho, 1991; Martins et al., 2011). Todos los procedimientos se hicieron conforme a las normas descritas por el Committee on Care and Use of Laboratory Animal Resources - National Research Council, USA (2011), para lo cual, los animales fueron tranquilizados con 2 -fenoxietanol $(300 \mathrm{mg} / \mathrm{L}$, Sigma Chemical Co, St. Louis) hasta observar pérdida del eje de nado y disminución de movimientos operculares, luego se procedió a efectuar un corte medular para su insensibilización.

\section{Procesamiento histológico y confrontación de resultados micro y macroscópicos}

Los ovarios fueron divididos en porciones craneal y distal, fijándose en formalina bufferada al 10\% por 24 horas y luego conservados en etanol al 70\%. Las muestras fueron enviadas para su respectivo procesamiento al Laboratorio de Histopatología de la empresa CORPAVET ${ }^{\circledR}$ en la ciudad de Bogotá (Colombia) en donde se incluyeron en parafina, con cortes de $5 \mu \mathrm{m}$ y coloreados con tinción de Hematoxilina - Eosina. Para la descripción de los hallazgos referentes a los estadios de desarrollo y estructura, las láminas se observaron en microscopio óptico Nikon Eclipse E400 ajustado a una cámara Nikon Digital Sihgt, DS-5M, Japón y el software Image ${ }^{\circledR}$ fue utilizado para el análisis de las imágenes. Fue empleada la clasificación de desarrollo oocitario sugerida por Landines (2005), sin embargo, para confrontar los hallazgos histológicos, fue necesario utilizar la escala de desarrollo macroscópico de Vazzoler (1996) para determinar el grado de madurez sexual en las hembras.

\section{Resultados}

Conforme a la clasificación macroscópica, los ovarios de $H$. marginatus presentaron dos estadios de desarrollo, siendo maduración (5 hembras) y madura (12 hembras) respectivamente, pese a lo anterior, los análisis histológicos permitieron encontrar en ambas fases todos los tipos celulares descritos a continuación:

Oocitos en cromatina nucleolar: Células esféricas caracterizadas por ser las más pequeñas de la línea germinativa, se encontraron formando pequeños grupos denominados "nidos" cuya cantidad varió entre 5 a 6 células. Tienen un núcleo redondo con poca afinidad tintoral y poseen desde 1 hasta 3 nucléolos. El citoplasma es escaso y levemente basófilo. El diámetro total de este tipo de células fue de 25,60 $\pm 2,70 \mu \mathrm{m}$ y su núcleo de 11,26 $\pm 1,07 \mu \mathrm{m}$ (Figura 2A).

Oocitos perinucleolares: Células germinativas mayores que las de la fase anterior y ya se observan de manera independiente, pues también se han desprendido de los nidos celulares. El núcleo es central, esférico y con mayor afinidad tintoral. De manera simultánea al crecimiento oocitario, aparecen numerosos nucléolos que alcanzan la periferia del núcleo. La envoltura folicular se presenta irregular y está constituida por dos membranas paralelas que se unen, siendo la membrana basal y una delgada teca conectiva. El diámetro total fue de $81,29 \pm 1,63 \mu \mathrm{m}$ y el núcleo de $51,22 \pm 1,36$ $\mu \mathrm{m}$ (Figura 2B).

Oocitos en fase de alvéolo cortical: El núcleo aparece con una cromatina menos condensada y con bastantes nucléolos acidófilos. Esta fase es caracterizada por el aparecimiento de alvéolos corticales que se forman en la periferia del citoplasma. En esta fase de desarrollo las células presentan un diámetro oocitario de 121,88 $\pm 1,38 \mu \mathrm{m}$ y un núcleo de $61,17 \pm 2,90 \mu \mathrm{m}$ (Figura $2 \mathrm{C}$ ).

Oocitos vitelogénicos: Estas células (184,94 \pm 2,65 $\mu \mathrm{m})$ presentan gránulos de vitelo acidófilos redondos con tamaños variados y que ocupan la mayoría del citoplasma. Presentan un núcleo central $(79,84 \pm 17,43$ $\mu \mathrm{m})$ con nucléolos en la periferia y la membrana basal se torna más delgada, alrededor de ella se denota la teca (Figura 2D). 
Oocitos maduros: Caracterizado por su aumento de tamaño $(311,30 \pm 16,37 \mu \mathrm{m})$ y morfológicamente por la clarificación del vitelo en virtud de la fusión de los gránulos de vitelo, al mismo tiempo, las concreciones lipídicas también son coalescentes. En este estudio, el núcleo (vesícula germinativa) no fue visible, pero normalmente, durante este estadio ya ha migrado desde la posición central hacia la periferia (Figura 2E).

Los diámetros de los folículos pos-ovulatorios (Figura 2F) no fueron medidos, debido a que estos no son estructuras totalmente esféricas. Además, teniendo como precedente el hallazgo de todos los tipos celulares con diámetros heterogéneos, sobre todo con prevalencia de oocitos en fase previtelogénica durante los meses de colecta, se puede inferir que $H$. marginatus es una especie asincrónica con desove parcial.

\section{Discusión}

En Colombia, la acuicultura continental ha dependido del cultivo de tres especies ícticas, siendo la tilapia roja y tilapia plateada (Oreochromis sp) las que más se producen y que equivalen al 51,8 y $13,5 \%$, la cachama blanca (Piaractus brachypomus) con 21,5\% y la trucha arco iris (Oncorhynchus mykiss) con 7,6\% (FEDEACUA, 2015). Ante esta situación, es claro que Colombia presenta una limitada oferta de especies ícticas nativas que son cultivables, por ende, y de acuerdo a las características que ofrecen los bagres, como la calidad de carne y fácil comercialización, es necesario implementar paquetes tecnológicos de especies con potencial productivo. El género Hypophthalmus se ha posicionado como una alternativa para la acuicultura, pero el conocimiento de su reproducción en sus cuencas de origen para su establecimiento en cautiverio aun es incipiente.

El estudio histológico del desarrollo oocitario en $H$. marginatus, permitió evidenciar la presencia de todas las células correspondientes a la oogénesis, nuestros resultados podrían compararse con los reportes obtenidos de estudios en otras especies pertenecientes a la familia Pimelodidae, siendo más específicamente en $H$. edentatus (Benedito-Cecilio y Agostinho, 1991) y Pseudoplatystoma magdaleniatum (Arce-Zúñiga et al., 2014). Con respecto al tipo de desove asincrónico, la capacidad de $H$. marginatus de mantener un stock de células de reserva, parece ser una estrategia para la supervivencia de la población. Según BrownPeterson et al., (2011) esta característica en algunas especies, les permitiría desoves sucesivos a lo largo de su ciclo de vida.

Este tipo de comportamiento, también fue determinado para $H$. marginatus pero en ejemplares nativos del río Tocantins (Brasil) (Aniceto-Cintra et al., 2008), por otra parte, existe la posibilidad no confirmada de que la misma especie posea los mismos hábitos reproductivos en el mismo río según los resultados de Hainfellner (2015), pues en este estudio, no fueron observados todos los tipos celulares, lo que impide deducir sobre su desove parcial. En otros Siluriformes, se evidenció a lo largo de un año de estudio, que especies como el Liso Rhamdia quelen en el río Sinú (Nieto et al., 2010) e Iheringichthys labrosus en el lago de Furnas (Minas Gerais, Brasil) (Santos et al., 2004) presentaran una actividad reproductiva constante y con este tipo de desove. No obstante, el número de puestas podría variar anualmente si el ecosistema no mantiene las mismas fluctuaciones alimenticias y el área de desove se encuentra en competencia con otras especies (Kramer, 1978).

\section{Agradecimientos}

Los autores agradecen a Rogelio Velásquez, Tilo y Frank Grimaldo, pescadores del municipio de Puerto Rico (Meta) por la captura de los ejemplares.

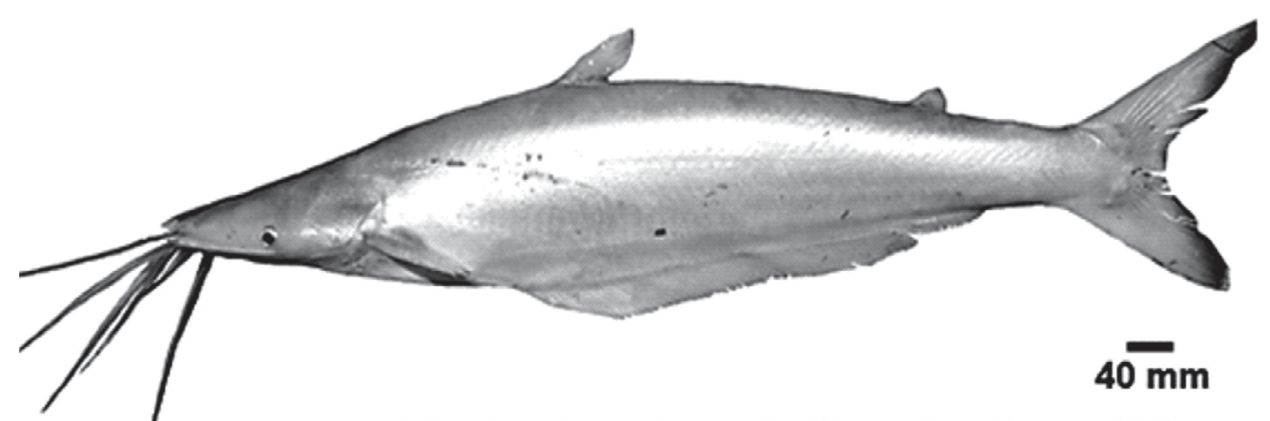

Foto: Jonathan Fernando Villamil Rodríguez, 2017.

Figura 1. Hembra sexualmente madura de mapará Hypophthalmus marginatus. 

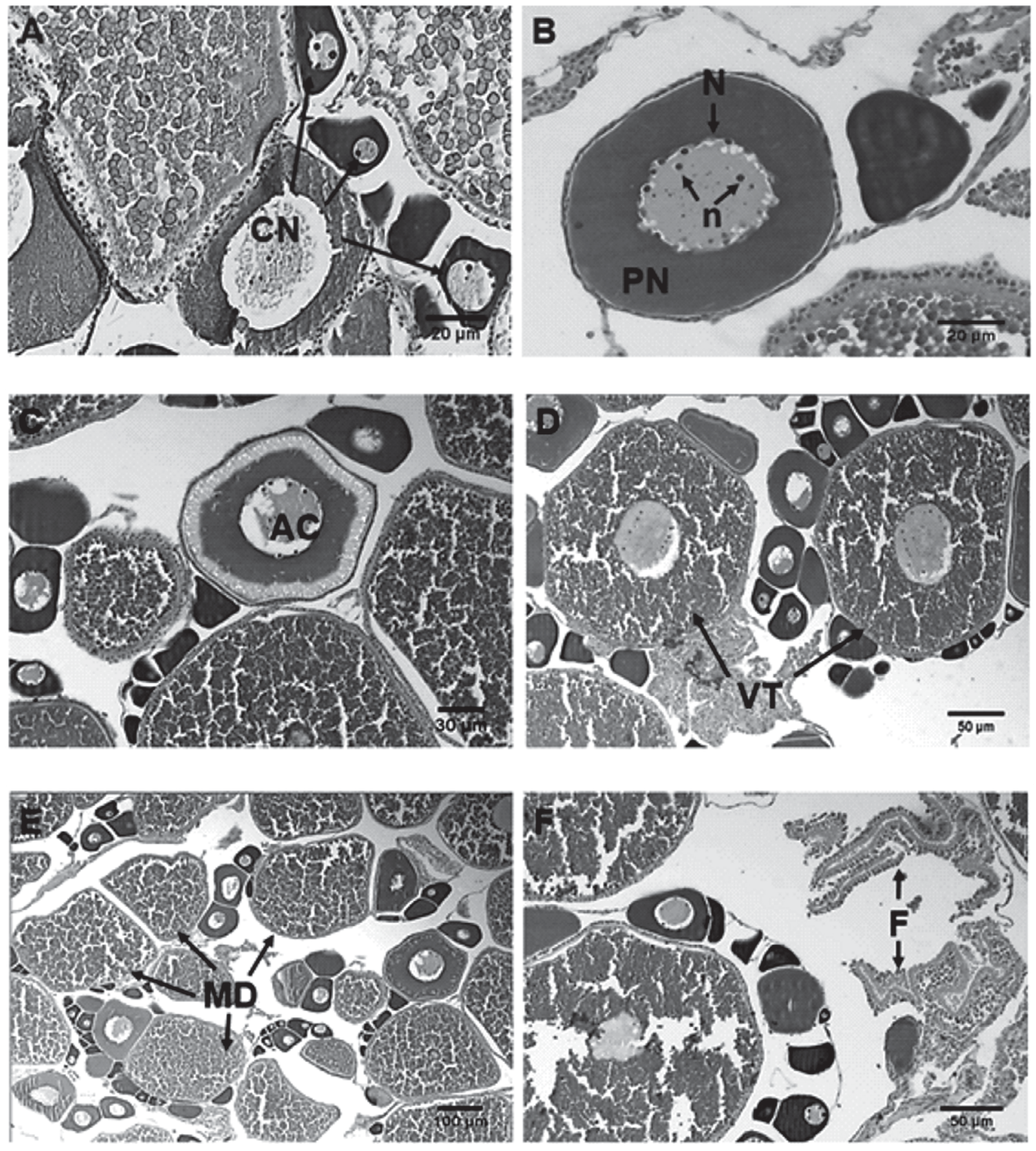

Figura 2. Caracterización de las células del linaje germinativo en hembras de $H$. marginatus. A. Oocitos en cromatina nucleolar (CN) 20x. B. Oocito perinucleolar (PN), Núcleo (N), nucléolos (n) 10x. C. Oocito en fase de alvéolo cortical (AC) 10x. D. Oocitos vitelogénicos (VT) 10x. E. Oocitos maduros (MD) 4x. F. Folículos pos-ovulatorios (F) 10x.

\section{Referencias}

Agostinho AA, Júlio JR, Petrere MJR. 1994. Itaipu reservoir (Brazil): impacts of the impoundment on the fish fauna and fisheries. In: I. G, Cowx. (Ed.). Rehabilitation of freshwater fisheries. London: Fishing New Books, p. 171-184.
Alcântara Neto CP. 1994. Ecologia da pesca dos maparás, Hypophthalmus spp. (Siluriformes, Hypophthalmidae), no lago Grande de Monte Alegre, Baixo Amazonas, Pará. Dissertação. Belém (PA): Universidade Federal do Pará, p. 141. 
Aniceto-Cintra IH, Carvalho da Rocha J, Juras A, Cutrim Souza R, Ogawa M. Biologia do mapará, Hypophthalmus marginatus (Valenciennes, 1840), no Reservatório da Usina Hidrelétrica de Tucuruí (Pará-Brasil). Boletím Técnico Científico do Cepnor. 2008;8(1):83-95.

Araújo-Lima CARM, Ruffino ML. 2004. Migratory Fishes of the Brazilian Amazon. En: Carolsfield J, Harvey B, Ross C, Baer A (Eds). Migratory Fishes of South America. Biology, Fisheries and Conservation Status. p. 168-221.

Arce-Zúñiga JW, Alonso-González JC, Hernández-Barrero S, Valderrama-Barco M. Determinación del tipo de desove y nivel de fecundidad del bagre rayado del Magdalena, Pseudoplatystoma magdaleniatum Buitrago-Suárez y Burr, 2007 (Siluriformes: Pimelodidae). Biota Colombiana. 2014;15(1):70-82.

Benedito-Cecilio E, Agostinho A. Biologia reprodutiva de Hypophthalmus edentatus (Spix, 1829) (Osteichthyes, Siluriformes) no reservatório de Itaipu - PR. II. Estrutura dos ovários e escala de maturidae. Revista UNIMAR. 1991;13(2):211-227.

Brown-Peterson N, Wyanski DM, Saborido-Rey F, Macewicz BJ, Lowerre-Barbieri SK. A standardized terminology for describing reproductive development in fishes. Mar Coast Fish. 2011;3(1):52-70

Carvalho ML, Goulding M. On the feeding ecology of the catfish Hypophthalmus fimbriatus in the blackwater Río Negro of the Amazon Basin. Rev Bras Zool. 1985; 35(1): 85-92.

Cutrim L, Batista VS. Determinação de idade e crescimento do mapará (Hypophthalmus marginatus) na Amazônia Central. Acta Amazônica. 2005;35(1):85-92.

Da Silva Ribeiro C, Moreira RG. Fatores ambientais e reprodução dos peixes. Revista da Biología. 2012;8:58-61.

FEDEACUA - Federación Colombiana de Acuicultores. Plan de Negocio Sectorial de la Piscicultura Colombiana, 2015; [0412-2018] URL: https://www.ptp.com.co/CMSPages/GetFile. aspx?guid=e4960689-709d-4fa6-9c62-d564782428f8

Ferraris-JR C. Checklist of catfishes, recent and fossil (Osteichthyes: Siluriformes), and catalogue of siluriform primary types. Zootaxa. 2007;1418:1-628.

Hainfellner P. 2015. Ciclo reprodutivo e indução hormonal de fêmeas de mapará (Hypophthalmus marginatus) no baixo Rio Tocantins, à jusante da barragem de Tucuruí. Tese (Doutorado) apresentada ao Programa de Pós-graduação em Aquicultura do Centro de Aquicultura da UNESP - CAUNESP, p. 103.

Hiramatsu N, Todo T, Sullivan CV, et al. Ovarian yolk formation in fishes: Molecular mechanisms underlying formation of lipid droplets and vitellogenin-derived yolk proteins. Gen Comp Endocrinol. 2015;221:9-15.
Honji RM, Guimarães Moreira R. Controle neuroendócrino da ovogênese em peixes teleósteos. Rev Bras Reprod Anim. 2017;41(1):86-93.

Kagawa H. Oogenesis in Teleost Fish. Aqua-BioScience Monographs. 2013;6(4):99-127.

Kramer DL. Reproductive seasonality in the fishes of a tropical stream. Ecology. 1978;59(5):976-985.

Landines MA. 2005. Mecanismos celulares de la reproducción de los peces. Capítulo I En: Daza PV, Landines PMA, Sanabria OAI. (Eds). 2005. Reproducción de Peces en el Trópico. Instituto Colombiano de Desarrollo Rural (INCODER) y Universidad Nacional de Colombia. Bogotá D.C., Colombia. p. 11-22.

López-Fernández H, Winemiller KO. A review of Venezuelan species of Hypophthalmus (Siluriformes: Pimelodidae). Ichthyol Explor Freshw. 2000;11:35-46.

Lowe-McConnell RH. 1987. Ecological studies in tropical fish communities. Cambridge (U. K.): Cambridge University Press, p. 382.

Martins J, Juras A, Araújo M, Mello Filho A, Cintra I. Seletividade da rede malhadeira-fixa para a captura do mapará, Hypophthalmus marginatus, no reservatório da usina hidrelétrica de Tucuruí, estado do Pará, Brasil. Bol Inst Pesca. 2011;37(2):123-133.

Mylonas CC, Fostier A, Zanuy S. Broodstock management and hormonal manipulations of fish reproduction. Gen Comp Endocrinol. 2010;165:516-534.

National Research Council. 2011. Guide for the Care and Use of Laboratory Animals: Eighth Edition. Washington, DC: The National Academies Press.

Nelson JS, Grande T, Wilson MVH. 2016. Fishes of the world. Fifth edition. John Wiley \& Sons, Hoboken, p. 707.

Nieto CWO, Rosso DFH, Pérez EEA. Biología reproductiva del Liso (Rhamdia quelen) en el río Sinú, Colombia. Acta Biolo Colomb. 2010;15(3):61-74.

Reis RE. Conserving the freshwater fishes of South America. International Zoo Yearbook. 2013;47(1):65-70.

Santos JE, Bazzoli N, Rizzo E, Santos GB. Reproduction of the catfish Iheringichthys labrosus (Lutken, 1874) (Siluriformes: Pimelodidae) in Furnas reservoir, Minas Gerais, Brazil. Rev Bras Zool. 2004;21:193-200.

Vazzoler AEA. 1996. Biologia da Reprodução de Peixes Teleósteos: Teoria e Prática. Nupelia, Maringá-Brasil. p. 169.

Zohar Y, Muñoz-Cueto J, Elizur A, Kah O. Neuroendocrinology of reproduction in teleost fish. Gen Comp Endocrinol. 2010;165(3):438-455. 